Classification

Physics Abstracts

$05.20-61.40 \mathrm{~K}$

\title{
Configuration of long chains in equilibrium with rings
}

\author{
R. G. Petschek \\ Department of Physics, Case Western Reserve University, Cleveland, Ohio 44106, U.S.A. \\ P. Pfeuty and J. C. Wheeler (*) \\ Laboratoire de Physique des Solides (**), Bâtiment 510, Université de Paris-Sud, 91405 Orsay Cedex, \\ France
}

(Reçu le 10 septembre 1984, accepté le 29 octobre 1984)

Résumé. - La distance $R$ entre deux monomères séparés par $N$ monomères sur une longue chaîne en équilibre chimique avec d'autres longues chaînes et des anneaux de toutes tailles varie suivant $R \sim N^{1 / D}$ avec $\frac{1}{D}=\frac{v(n)}{\phi(n)}$ où $v(n)$ et $\phi(n)$ sont respectivement l'exposant critique pour la longueur de corrélation et l'exposant de crossover lié à l'anisotropie quadratique pour le modèle $O(n)$ où $n$ est un poids statistique associé à un anneau.

Abstract. - The distance $R$ spanned by $N$ monomers of a long chain in chemical equilibrium with other long chains and rings of all sizes varies like $R \sim N^{1 / D}$ with $1 / D=\frac{v(n)}{\phi(n)}$ where $v(n)$ and $\phi(n)$ are respectively the correlation length critical exponent and the quadratic anisotropy crossover exponent of the $O(n)$ model with $n$ being a statistical preweighting factor associated with a ring.

For a long linear polymer of $N$ monomers, the mean size $R$ (end to end distance or radius of gyration) varies as :

$$
R \sim N^{1 / D}
$$

where the exponent $D$ plays the role of a fractal or Hausdorff dimension [1]. Three different such behaviours (universality classes) are known at present.

(i) Random walk behaviour : when no crossing restrictions are imposed, that is when interactions between monomers are limited to monomers that are nearly adjacent on the chain, it has been shown [2] that the exponent $D^{-1}$ is equal to $1 / 2$, independent of the spatial dimension $d$.

(*) Present and Permanent address : Dept. of Chemistry B-014, University of California, San Diego, Ca 92093, U.S.A.

$\left.{ }^{* *}\right)$ Laboratoire associé au C.N.R.S. 
(ii) Excluded volume behaviour : when excluded volume effects are present (whether due to genuine chain excluded volume or to statistical solvent effects due to a good solvent) the chain is swollen. The exponent $D^{-1}$ is larger than $1 / 2$ and depends [3] on the spatial dimension $d$. It has been shown [4] that $D^{-1}$ is equal to $v(n)$, the correlation length critical exponent of the $O(n)$ model in the limit $n \rightarrow 0$,

$$
D^{-1}=v(n=0) \text {. }
$$

Renormalization group (RG) calculations of the $O(n)$ field theory model [5] give an estimate of $D^{-1}=0.59$ in $d=3$ dimensions. In $d=2$ a Coulomb gas description of a $O(n)$ lattice model based on RG and universality assumptions leads [6] to $D^{-1}=3 / 4$. For $d>d_{\mathrm{c}}=4, D^{-1}$ sticks at the random walk value $1 / 2$.

(iii) Polyelectrolyte behaviour : for charged polymers with long range Coulomb interactions between monomers, $D^{-1}$ takes a larger value which depends [7] on $d$. For $d=3$, it is believed that $D^{-1}=1$ and it can be shown that $D^{-1}=1 / 2$ for $d>d_{\mathrm{c}}=6$.

The behaviours (ii) and (iii) are obtained for long isolated chains or for chains in solutions on a length scale $R \lesssim \xi$ where $\xi$ is the correlation length of the monomer-monomer correlation function which also measures the mean distance separating encounters between distinct chains. For $R \gg \xi$ the chains behave as random walks $[7,8]$. The random walk behaviour is also obtained for polymers in a poor solvent at the theta point [9], in 3 and more spatial dimensions.

Chemical equilibrium between monomers and polymers can also be described in terms of the $O(n)$ model. Recently we have shown that the equilibrium between monomers and linear chains is described [10] by the $n \rightarrow 0$ limit of the $n$-vector model. Under certain circumstances linear chains can close to form rings. For equilibrium polymerization of chains and rings it has been shown recently [11-14] that the critical nature of the transition is the same as for the $O(n)$ model, where $n$ is the statistical preweighting factor associated with a ring. In the case of liquid sulfur to which the theory of equilibrium polymerization is well suited, $n$ is known to be equal to unity $[11,13,14]$; no ring at all [10] corresponds to $n=0$; and directed rings [12], in some circumstances, correspond to $n=2$. It might be tempting to conjecture that in analogy to the case of chains alone the exponent $D^{-1}$ for a chain in the middle of rings is equal to the critical exponent $v(n)$ of the $O(n)$ model. However, we have also shown [15] that a second exponent, the crossover exponent for quadratic anisotropy $\phi(n)$ of the $O(n)$ vector model, plays a role in the description of the transition. Close to the polymerization transition $T_{\mathrm{p}}$ in the polymerized phase the concentration $\phi_{\mathrm{c}}$ of monomers incorporated in very large chains varies as $[15,16]$ :

$$
\phi_{\mathrm{c}} \sim\left|T-T_{\mathrm{p}}\right|^{[2-\alpha(n)-\phi(n)]}
$$

where $\alpha(n)$ is the "specific heat " exponent of the $O(n)$ vector model and is usually related (at least for $d<4)$ to $v(n)$ through the hyperscaling law :

$$
2-\alpha(n)=d v(n) .
$$

This suggests that $D^{-1}$ may be related both to $v(n)$ and $\phi(n)$. We argue in this Letter that the behaviour of chains in the midst of rings of all sizes is given by

$$
D=\phi(n) / v(n)
$$

so that $D$ is a function of both $d$ and $n$.

We give a simple heuristic argument which can be easily presented. This result (Eq. (5)) can also be obtained more rigorously within the framework of scaling theory from the monomer-monomer correlation function [16]. 
In the polymerized phase, for equilibrium polymerization close to $T_{\mathrm{p}}$, the system is represented schematically in figure 1 . Very long chains are in the midst of rings of all sizes. The mean distance separating encounters between distinct chains is proportional to the correlation length $\xi$ of the $O(n)$ model which varies as :

$$
\xi \sim\left|T-T_{\mathbf{p}}\right|^{-v(n)} .
$$

The mean density of monomers in chains $\phi_{\mathrm{c}}$ can be obtained by considering one chain for $R \lesssim \xi$ (since, on this length scale, the probability of encountering more than one chain is small). Let us suppose that on that length scale the distance $R$ between two monomers separated by $N$ monomers is :

$$
R \sim N^{1 / D}
$$

On this same distance scale $\xi$ the chain contains $N^{*} \sim \xi^{D}$ monomers and the density of monomers is given by :

$$
\phi_{\mathrm{c}} \sim \frac{N^{*}}{\xi^{d}} \sim \xi^{D-d}
$$

Combining equations (8) and (6) and using the hyperscaling law (4) and the known behaviour of $\phi_{\mathrm{c}}$ (Eq. (3)) leads to the result (Eq. (5)) relating $D$ to the critical exponents $v(n)$ and $\phi(n)$.

It can be shown that both equations (5) and (3) are valid for any dimension [16] although the hyperscaling law (4) is not valid for $d>d_{\mathrm{c}}=4$.

For $d>d_{\mathrm{c}}=4, \alpha(n)=0, v(n)=1 / 2, \phi(n)=1$ and thus $\phi_{\mathrm{c}} \sim \Delta T_{\mathrm{p}}$ and $D=2$. For $d=3$ the values of $v(n), \phi(n)$ and thus $D^{-1}(n)$ are known only approximately. The values are shown in table I for $n=0,1,2,3, \infty$. For $v(n)$ we quote the values obtained from field theory RG [5]. For $\phi, \phi(2)$ and $\phi(3)$ are taken from high temperature series expansions [17] and $\phi(1)$ is estimated from $\varepsilon$ expansions [17], and comparison with $\phi(2)$ and $\phi(3)$. For $n=0, \phi(0)=1$ and equation (2) is recovered. For $n \rightarrow \infty$, the $O(n)$ model behaves like the spherical model [18, 19] and $v(\infty)=\frac{1}{d-2}, \phi(\infty)=\frac{2}{d-2}$ and $D^{-1}(\infty)=1 / 2$ which is the random walk value. When $n$

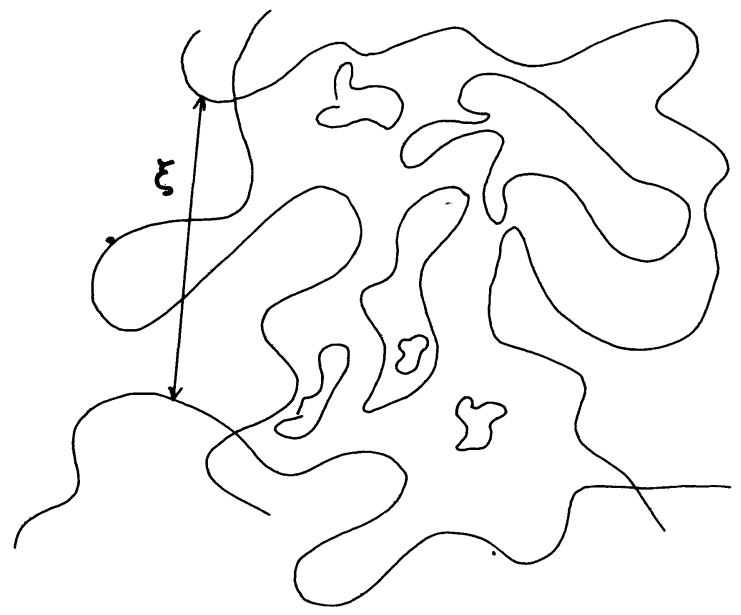

Fig. 1. - Schematic representation of the polymerized phase for equilibrium polymerization of chains and rings. 
Table I. - Approximate or exact values of $v(n), \phi(n)$ and $D^{-1}(n)$ in $d=3$ dimensions.

\begin{tabular}{|c|l|l|c|}
\hline \multicolumn{5}{|c|}{$d=3$} \\
\hline$n$ & $v(n)$ & $\phi(n)$ & $D^{-1}(n)=\frac{v(n)}{\phi(n)}$ \\
\hline 0 & 0.588 & 1 & 0.588 \\
1 & 0.63 & 1.1 & 0.573 \\
2 & 0.669 & 1.175 & 0.569 \\
3 & 0.705 & 1.25 & 0.564 \\
$\infty$ & 1 & 2 & 0.5 \\
\hline
\end{tabular}

varies from zero to infinity $D^{-1}$ varies from 0.59 to 0.5 . In two dimensions $v(0)$ has been estimated to be [6] $3 / 4$ and $\phi(0)=1$. The exact solution for the Ising model gives $v(1)=1$; the value of $\phi(1)$, however, is not known. Along the line of critical points for $n=2, D^{-1}$ can be found [20]. It varies from $2 / 3$ at the vortex unbinding transition to $1 / 2$ at zero temperature.

It is interesting to note that the argument given above for $D=\phi / v$ fails in more than four dimensions, even though the result is valid for all $d$. If one inserts the exponent values for $\alpha, \phi$ and $v$ into equation (8) for $d>4$ one finds $D=d-2$ rather than $D=2$. The error arises in identifying the density of monomers on a given chain polymer known to be in the vicinity with the total density of monomers in chain polymers for $d>4$. This is a good approximation for $d<4$ where the excluded volume effects have a profound effect both on the monomer-monomer correlation function and in excluding other polymers from the vicinity of a given polymer. For $d>4$ however, the polymers have Hausdorff dimensionality so much lower than that of the space in which they are embedded that the interactions leading to excluded volume have little effect either on the geometry of the polymer or in excluding other polymers from its vicinity. Thus the first approximate equality in equation (8) fails as well as equation (4). As noted above (5) may be obtained independently of dimensionality $d$ by consideration of scaling properties of the monomer-monomer correlation function. That argument will be presented in detail elsewhere [16].

One also sees from such an argument that the same behaviour is to be expected for the Hausdorff dimension of a very large ring in equilibrium with rings of all sizes, and holds very close to the transition temperature both above and below the transition temperature, that is, both in the presence and absence of large chains.

To the best of our knowledge, this is the first time that the fractal dimension of a polymer has been shown to depend upon a parameter ( $n$ ) different from the spatial dimension [21]. As is the case for the spatial dimensionality, however, it appears likely that the physically significant values of the parameters $n$ are discrete and integers.

\section{Acknowledgments.}

Research supported in part by NSF grants CHE 8119247 and INT 8212577 . One of the authors (JCW) gratefully acknowledges the support of a John Simon Guggenheim foundation fellowship. The hospitality of the Université de Paris-Sud, Centre d'Orsay, is gratefully acknowledged by JCW and RGP. 


\section{References}

[1] Mandelbrot, B. B., The fractal geometry of nature (Freeman, San Francisco, California) 1982.

[2] KAC, M., Am. Math Monthly, Vol. 54, 1946.

[3] FloRy, P. J., Principles of Polymer Chemistry (Cornell University Press, New York) 1953.

[4] De Gennes, P. G., Phys. Lett. A 38 (1972) 339.

[5] Le Guillou, J. C. and Zinn-Justin, J., Phys. Rev. B 21 (1980) 3976.

[6] Nienhuis, B., Phys. Rev. Lett. 49 (1982) 1062.

Nienhuis, B., J. Stat. Phys. 34 (1984) 731.

[7] De Gennes, P. G., Pincus, P., Velasco, R. M., Brochard, F., J. Physique 37 (1976) 1461. Pfeuty, P., Velasco, R., De Gennes, P. G., J. Physique Lett. 38 (1977) L-5. BAumgärtner, A., J. Physique Lett. 45 (1984) L-515.

[8] DaOud, M., Macromolecules 8 (1975) 804.

De Gennes, P. G., Riv. del Nuovo Cimento 7 (1977) 363.

[9] De Gennes, P. G., J. Physique Lett. 36 (1975) 55.

[10] Wheeler, J. C., Kennedy, S. J., Pfeuty, P., Phys. Rev. Lett. 45 (1980) 1748.

[11] CORdery, R., Phys. Rev. Lett. 47 (1981) 457.

[12] Helfrich, W. and Müller, W., in Continuous Models of Discrete Systems (Univ. of Waterloo Press, Waterloo, Ontario) 1980, p. 753.

[13] Pfeuty, P. and Wheeler, J. C., Phys. Lett. A 84 (1981) 493.

[14] Duplantier, B. and Pfeuty, P., J. Phys. A 15 (1982) L-127.

[15] Wheeler, J. C., Petschek, R. G., Pfeuty, P., Phys. Rev. Lett. 50 (1983) 17.

[16] Petschek, R. G., Pfeuty, P., Wheeler, J. C., in preparation.

[17] Pfeuty, P., Jasnow, D., Fisher, M., Phys. Rev. B 10 (1974) 2088.

[18] Stanley, H. E., Phys. Rev. 176 (1968) 718.

[19] RIEDel, E. K. and Wegner, F. J., Z. Phys. 225 (1969) 195.

[20] José, J. V., Kadonoff, L. P., KirkPatrick, S., Nelson, D. R., Phys. Rev. B 16 (1977) 1217.

[21] For percolation there is an analogous situation with an infinite cluster appearing for $p>p_{\mathrm{c}}$. The transition is described by the $s$ states $s \rightarrow 1$ Potts model and the fractal dimension of the infinite cluster $D=\frac{\Delta(s=1)}{v(s=1)}$ where $\Delta$ is the crossover exponent of the $s \rightarrow 1$ Potts model associated with the « magnetic " field. See Aharony, A., J. Stat. Phys. 34 (1984) 931. 